\title{
Multi-objective optimization of the streamlined head of high-speed trains based on the Kriging model
}

\author{
YAO ShuanBao ${ }^{1}$, GUO DiLong ${ }^{1}$, SUN ZhenXu ${ }^{1 *}$, YANG GuoWei $^{1} \&$ CHEN DaWei ${ }^{2}$ \\ ${ }^{1}$ Key Laboratory for Application of Mechanics to Engineering Systems, Institute of Mechanics, Chinese Academy of Sciences, \\ Beijing 100190, China; \\ ${ }^{2}$ CSR Qingdao Sifang Locomotive Co., Ltd., Qingdao 266111, China
}

Received June 13, 2012; accepted August 24, 2012; published online October 1, 2012

\begin{abstract}
As the running speed of high-speed trains increases, aerodynamic drag becomes the key factor which limits the further increase of the running speed and energy consumption. Aerodynamic lift of the trailing car also becomes the key force which affects the amenity and safety of the train. In the present paper, a simplified CRH380A high-speed train with three carriages is chosen as the model in order to optimize aerodynamic drag of the total train and aerodynamic lift of the trailing car. A constrained multi-objective optimization design of the aerodynamic head shape of high-speed trains based on adaptive non-dominated sorting genetic algorithm is also developed combining local function three-dimensional parametric approach and central Latin hypercube sampling method with maximin criteria based on the iterative local search algorithm. The results show that local function parametric approach can be well applied to optimal design of complex three-dimensional aerodynamic shape, and the adaptive non-dominated sorting genetic algorithm can be more accurate and efficient to find the Pareto front. After optimization the aerodynamic drag of the simplified train with three carriages is reduced by $3.2 \%$, and the lift coefficient of the trailing car by $8.24 \%$, the volume of the streamlined head by $2.16 \%$; the aerodynamic drag of the real prototype CRH $380 \mathrm{~A}$ is reduced by $2.26 \%$, lift coefficient of the trailing car by $19.67 \%$. The variation of aerodynamic performance between the simplified train and the true train is mainly concentrated in the deformation region of the nose cone and tail cone. The optimization approach proposed in the present paper is simple yet efficient, and sheds lights on the constrained multi-objective engineering optimization design of aerodynamic shape of high-speed trains.
\end{abstract}

multi-objective optimization, Kriging, genetic algorithms, aerodynamic shape, high-speed trains

Citation: Yao S B, Guo D L, Sun Z X, et al. Multi-objective optimization of the streamlined head of high-speed trains based on the Kriging model. Sci China Tech Sci, 2012, 55: 3495-3509, doi: 10.1007/s11431-012-5038-8

\section{Introduction}

The development of high-speed train technology is an important sign of a national high-tech development. In recent years, China's high-speed train technology has made significant progress. The operating speed of Beijing-Shanghai high-speed rail has been up to $300 \mathrm{~km} \mathrm{~h}^{-1}$. In the case of high-speed operation, the aerodynamic characteristics of

*Corresponding author (email: sunzhenxu@imech.ac.cn) high-speed trains become more complex, since high-speed trains travel close to the ground and their aspect ratio is much larger than other transports [1-3]. Resistance characteristics of the trains are directly related to their speed and the ability of energy saving and environmental protection. The aerodynamic drag of high-speed trains whose streamlined head is slightly larger than $5 \mathrm{~m}$ at the speed of $300 \mathrm{~km} \mathrm{~h}^{-1}$ is up to $85 \%$ of the total drag. Meanwhile, the aerodynamic drag of high-speed trains whose streamlined head is about $10 \mathrm{~m}$ is up to $75 \%$ of the total drag [4] at the speed of $300 \mathrm{~km} \mathrm{~h}^{-1}$. In conditions without crosswind the aerodynamic lift of the 
trailing car is the key force which affects the amenity and safety of the train. It has an adverse effect on the smooth travelling and comfort of the trailing car that aerodynamic uplift force will significantly reduce the wheel-rail contact force. Meanwhile, excessive uplift force easily leads to derailment so that operational safety is badly affected. Therefore, to reduce aerodynamic drag of the train and aerodynamic lift of the tailing car becomes the key issue of optimal design of head shape of high-speed trains. The head of a high-speed train has complex streamlined shape, and its design parameters are as many as dozens. Currently, the main design method of the shape of high-speed trains is to obtain individual key parameters and their relationship with aerodynamic performance of the trains by traditional wind tunnel tests, dynamic model tests and real vehicle tests, and then to improve the design appropriately. But this method suffers from large investment and long design cycles. With the gradual development of computer technology and the engineering application of CFD (computational fluid dynamics), CFD has been successfully applied to the design and study of high-speed trains. Yet in spite of this, the design of the shape of a high-speed train with a good aerodynamic performance still needs to perform a large number of calculations and costs a lot of computing resources. Moreover, the relationship obtained is often only the one between single design parameter and optimization goal.

At present, there are few references on multi-objective optimization design of the aerodynamic shape of the highspeed train using both CFD and optimization algorithm. The existing research [5-10] mostly focuses on two-dimensional contour of trains or unconstrained single-objective optimization with less efficient optimization algorithm, and is difficult to be applied to practical engineering problems with constraints, large amounts of computation and a number of design parameters. Ku et al. [5] combined two single-objective optimization processes, obtained the optimal rate of section change of streamlined parts of the leading car having micro-pressure waves as a goal which were generated through tunnels, and then completed the single-objective optimization design based on Kriging model and VMF (Vehicle Modeling Function) three-dimensional parametric approach to reduce aerodynamic drag of the leading car on the premise that the rate of section change is constant. Combining genetic algorithms and arbitrary mesh deformation technique, Sun et al. [6] extracted four design parameters and used the unconstrained single-objective optimization design aiming at the aerodynamic drag of the streamlined head of CRH3 leading car. In the optimization process, the flow field was calculated for 96 times using 6 generations of genetic algorithm, and the nonlinear relationship of the design variables and optimization goals was obtained using Kriging model. In order to reduce the intensity of micro-pressure waves, unconstrained single-objective optimization design for the area change ratio of the streamlined head with different nose cone lengths based on BFGS
(Broyden-Fletcher-Goldfarb) algorithm and response surface model was studied in ref. [7] by Ku et al. Based on support vector machine model and continuous quadratic programming method, Lee et al. [8] extracted 9 design variables, designed 100 experimental sample points for train longitudinal profile lines, and used single-objective optimization design of the train aerodynamic shape to reduce the intensity of micro-pressure waves. In the present paper, on the basis of the above literature, the local function parametric approach based on free form deformation is used for CRH380A high-speed train streamlined shape. Central Latin hypercube sampling method with maximin criteria based on the iterative local search algorithm is adopted to reduce aerodynamic drag of the whole train, aerodynamic lift of the trailing car with 5 key design variables extracted and 16 sample points designed. Kriging surrogate model which meets the requirement of engineering precision is designed after 4 times iterations by using single-objective optimal design based on minimizing the response surface criteria and optimizing the relevant parameters of the Kriging surrogate model with adaptive genetic algorithm. Combined with adaptive non-dominated sorting genetic algorithm, a multi-objective constrained efficient aerodynamic shape optimization method is developed in the present paper. According to this method, Pareto optimal solutions is obtained. After that, aerodynamic performance of the optimized shape and the practical shape of CRH380A with three carriages is comparatively analyzed.

\section{Kriging surrogate model}

The Kriging surrogate model is an interpolation technique based on statistical theory [11-14]. This model takes full account of the relevant characteristics of the variable space, containing the regression part and the nonparametric part:

$$
y\left(\boldsymbol{x}^{(i)}\right)=F^{T}\left(\boldsymbol{x}^{(i)}\right) \beta+z\left(\boldsymbol{x}^{(i)}\right),(i=1, \cdots, n),
$$

where $n$ is the number of sample points, $\beta$ is the regression coefficient, $\boldsymbol{x}^{(i)}$ is sample points and $F^{T}\left(\boldsymbol{x}^{(i)}\right)$ is deterministic function that is a global approximation of the design space represented by the polynomial of $x$. In this paper, regression part $F^{T}\left(\boldsymbol{x}^{(i)}\right)$ is taken as constant $\mu$ because the random part has the ability to capture the trend of response objects [15, 16]. $z\left(\boldsymbol{x}^{(i)}\right)$ is the error of random distribution and could provide the approximation of the local deviation. Its mean is zero, its variance is $\sigma_{z}^{2}$ and its covariance matrix takes the form as follows:

$$
\operatorname{cov}\left[z\left(\boldsymbol{x}^{(i)}\right), z\left(\boldsymbol{x}^{(j)}\right)\right]=\sigma_{z}^{2}\left[R\left(\theta, \boldsymbol{x}^{(i)}, \boldsymbol{x}^{(j)}\right)\right],\left(i, j=1, \cdots, n_{\mathrm{s}}\right),
$$

where $n_{\mathrm{s}}$ is the number of training sample points, $R\left(\theta, \boldsymbol{x}^{(i)}\right.$, $\left.\boldsymbol{x}^{(j)}\right)$ is the correlation function with parameter $\theta$ represent- 
ing the spatial correlation among the training sample points. It uses Gaussian function as the correlation function, and its expression is

$$
R\left(\theta, \boldsymbol{x}^{(i)}, \boldsymbol{x}^{(j)}\right)=\exp \left[-d\left(\boldsymbol{x}^{(i)}, \boldsymbol{x}^{(j)}\right)\right]
$$

where,

$$
d\left(\boldsymbol{x}^{(i)}, \boldsymbol{x}^{(j)}\right)=\sum_{k=1}^{m} \theta_{k}\left|x_{k}^{(i)}-x_{k}^{(j)}\right|^{2} ;\left(i, j=1, \cdots, n_{\mathrm{s}}\right),
$$

$M$ is the number of design variables, and $x_{k}^{(i)}$ is the value of the design of the sample points corresponding to sample point $\boldsymbol{x}^{(i)}$. When the values of $\theta_{k}$ are the same, the related function is isotropic, that is, all the components of sample point $\boldsymbol{x}^{(i)}$ have the same weight. In this situation, the prediction accuracy of the model will be reduced. Therefore, the values of $\theta_{k}$ are different in this paper in order to increase the prediction accuracy of Kriging model.

To improve the prediction accuracy and the generalization ability of the model, the mean of prediction error must be zero, and the mean square error of prediction error must be the minimum, and then the concentrated log-likelihood function will be obtained as follows:

$$
-\frac{n_{\mathrm{s}}}{2} \ln \left(\hat{\boldsymbol{\sigma}}_{z}^{2}\right)-\frac{1}{2} \ln (|\boldsymbol{R}|)
$$

By solving the maximization problem of eq. (5),

$$
\max _{\theta_{k}>0}\left(-\frac{n_{s}}{2} \ln \left(\hat{\boldsymbol{\sigma}}_{z}^{2}\right)-\frac{1}{2} \ln (|\boldsymbol{R}|)\right), k=1, \cdots m .
$$

The values of $\theta_{k}$ will be obtained.

The response value of any point within the design space can be estimated by a linear combination of the response value of training sample points, then the mean square error of the prediction response value can be given by

$$
\begin{gathered}
\hat{\boldsymbol{y}}\left(\boldsymbol{x}^{*}\right)=\hat{\boldsymbol{\mu}}+\boldsymbol{r}\left(\boldsymbol{x}^{*}\right)^{T} \boldsymbol{R}^{-1}(\boldsymbol{Y}-\boldsymbol{1} \hat{\boldsymbol{\mu}}), \\
\boldsymbol{\sigma}^{2}\left(\boldsymbol{x}^{*}\right)=\boldsymbol{\sigma}_{z}^{2}\left(\boldsymbol{I}+\left(\boldsymbol{1}^{T} \boldsymbol{R}^{-1} \boldsymbol{l}\right)^{-1}\left(\boldsymbol{I}-\boldsymbol{I}^{T} \boldsymbol{R}^{-1} \boldsymbol{r}\right)^{2}-\boldsymbol{r}^{T} \boldsymbol{R}^{-1} \boldsymbol{r}\right),
\end{gathered}
$$

where,

$$
\begin{gathered}
\hat{\boldsymbol{\mu}}=\left(\boldsymbol{I}^{T} \boldsymbol{R}^{-1} \boldsymbol{I}\right)^{-1} \boldsymbol{I}^{T} \boldsymbol{R}^{-1} \boldsymbol{Y}, \\
\hat{\boldsymbol{\sigma}}_{z}^{2}=\frac{(\boldsymbol{Y}-\boldsymbol{1} \hat{\boldsymbol{\mu}})^{T} \boldsymbol{R}^{-1}(\boldsymbol{Y}-\boldsymbol{1} \hat{\boldsymbol{\mu}})}{n_{\mathrm{s}}},
\end{gathered}
$$

$\boldsymbol{I}$ is the matrix of which all elements are $1, \boldsymbol{Y}$ is the response value of training sample points, and $\boldsymbol{r}$ is the vector of correlation function between $\boldsymbol{x}^{*}$ and the initial training sample points.

\section{Local shape function parametric approach}

Geometry parametric approach plays an important role in aerodynamic optimal design. An efficient parametric method can not only describe the changes of shape completely, but also can reduce the optimization cycle and improve optimization efficiency. The performance of the FFD method [17] and NURBS method is perfect, which can control large deformation regions with a few control points and ensure the smoothness of surface. But for three-dimensional parameterization of the complex surface, they are difficult for application because practical engineering optimization problems require more design variables and large computation cost. In order to solve this problem, Kulfan et al. [18] proposed the class and shape function parametric method and realized the $2 \mathrm{D}, 3 \mathrm{D}$ parametric designs of the airfoil, wing and fuselage. On this basis, Rho et al. [19] proposed the VMF 3D parametric approach, and used less design parameters to realize 3D parametric designs of the complex shape of the automobiles. Ku et al. [5] applied VMF method to 3D parametric design of the streamline of high-speed trains, and the drag reduction of the streamline was successfully conducted. In this paper, a new parametric approach called Local Shape Function (LSF) which is based on FFD method and NURBS method has been designed.

The whole processes are as follows:

(1) For a given geometry, deformation regions should be divided firstly.

(2) Mesh the deformation regions, and obtain the coordinate values of every grid point. In order to keep the smooth transition of the surface, the structural grids have been utilized for mesh discretization, just as Figure 1 shows.

(3) Choose the deformation function of each region, which can be selected randomly, but smooth transition between adjacent regions should be ensured.

(4) Choose a weight factor $W_{i}$ for each deformation function, which determines the maximum deformation value of each region.

(5) Calculate the increments $\Delta$ of coordinates of all grid points by the deformation functions and $W_{i}$.

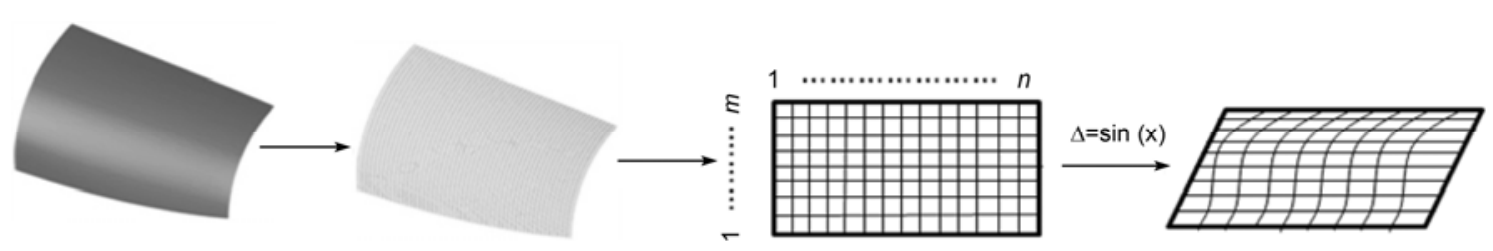

Figure 1 Schematic of surface deformation by LSF. 
(6) Get the coordinates of the deformed shape by sum$\operatorname{ming} \Delta$ and the coordinates of the original shape.

(7) According to the coordinates of the deformed shape, the deformed surface can be fitted exactly, then a deformation process is done.

In the above process, step (3) is the most crucial step. The deformation surfaces are different from each other due to the different choices of deformation functions. Inappropriate deformation functions will easily lead to irrational deformation surfaces. Trigonometric functions, exponential functions, logarithmic functions, polynomial functions and NURBS functions are all commonly used deformation functions.

Due to the symmetrical design along the longitude of the train, only one side of the symmetrical plane of the streamline is parameterized. As a result, the design parameters can be reduced by half. The parametric surfaces are separated into 7 deformation regions, as shown in Figure 2(a). Nose drainage is controlled by zones 3 and 6 , and a control point Pointl is set here, extracting its $y$ coordinate as the first design parameter $w_{1}$. Nose height is controlled by zones 1 and 3 , and another control point Point 2 is set here, extracting its $z$ coordinate as the second design parameter $w_{2}$. The width of the streamline is controlled by zones $4-7$, and a control point Point 3 is set here, extracting its $y$ coordinate as the third design parameter $w_{3}$. The slope of the cab window is controlled by zones 5 and 6 , and the fourth control point Point4 is set here, extracting its $z$ coordinate as the fourth design parameter $w_{4}$. The shape of the cowcatcher is controlled by zone 2, and the fifth control point Point 5 is set here, extracting its $x$ coordinate as the fifth design parameter. Except for the zone around the cowcatcher, the width of the bottom of the streamline will not deform so as to contain the ancillary components. For simplicity, all the deformation functions in this paper are trigonometric functions. Figure 2(b) shows the deformation of the nose and cowcatcher. It can be seen that the deformation method can ensure the surface smoothness and smooth transition among different deformation regions.

\section{CFD algorithms and accuracy validation}

\subsection{Computational model}

Optimal head design of high-speed trains mainly focuses on the streamlined parts, of which the key design parameters are: the cross-sectional area distribution of the nose of the high-speed trains, the slenderness ratio of the streamlined head, the longitudinal-type line and horizontal-type line of the streamlined head, nose drainage, cab perspective and the side hood of bogie. The model used for shape optimization is a simplified prototype of CRH380A, which consists of a leading car, a middle car and a rear car. The shield is completely closed and the cavity around the bogie zone is closed too. The bogie beneath the streamline of the trailing car would fiercely affect the wake flow, so that this bogie is considered in the present paper so as to better predict the lift of the trailing car, just as Figure 3 shows.

The geometric parameters of the simplified shape of CRH380A are as follows: the length of the leading car and trailing car is $26.5 \mathrm{~m}$, and the shape of the rear car is the same as the leading car. The length of the middle car is $25 \mathrm{~m}$. The length of the streamlined head is $12 \mathrm{~m}$, the height is $3.5 \mathrm{~m}$, the width is $3.38 \mathrm{~m}$, cross-sectional area is $11.2 \mathrm{~m}^{2}$ and the slenderness ratio is 3.55 . The type of the cross-sectional area distribution of CRH380A is a three-sectional type, of which the longitudinal-type line is double-arch, the horizontal-type line is flat spindle and the nose is of ellipsoid, just as shown in Figure 3.

\subsection{CFD algorithm}

CFD accuracy that directly affects the construction of the response surface function and efficiency of optimization algorithm is the basis for the whole optimization process. In this paper, the speed of high-speed train is $300 \mathrm{~km} \mathrm{~h}^{-1}$, so the Mach number is 0.245 . Under this condition, the air compression characteristic has an obvious effect on the aerodynamic drag of the train. Therefore, the steady compressible Reynolds-averaged Navier-Stokes equations [20]

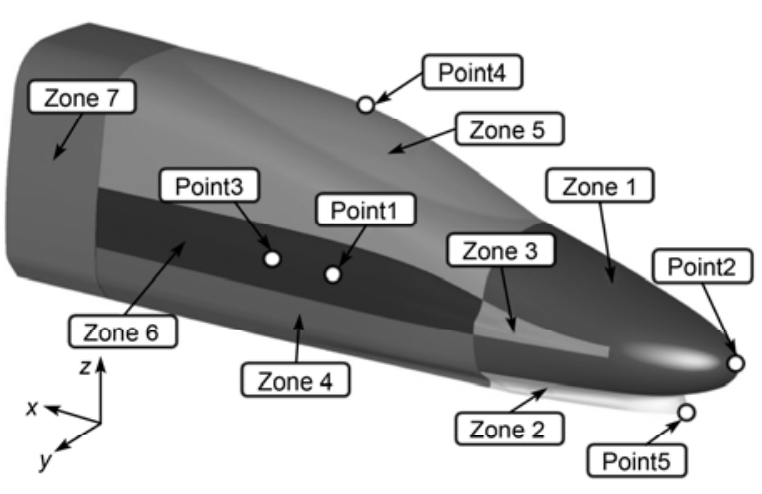

(a)

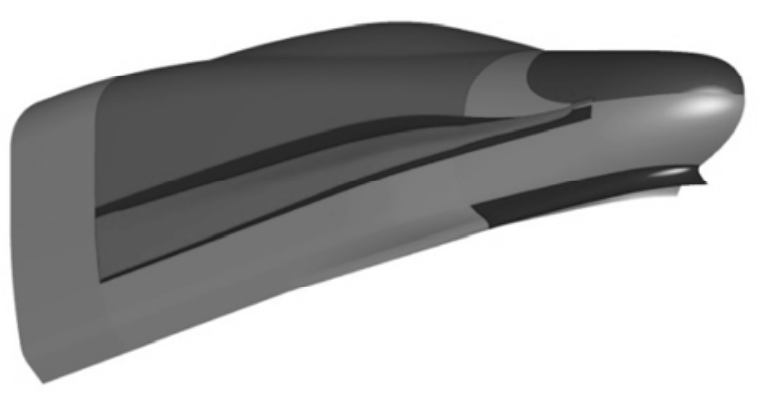

(b)

Figure 2 Deformation zones and local deformations. (a) Schematic of deformation zones; (b) deformation of the nose and cowcatcher. 

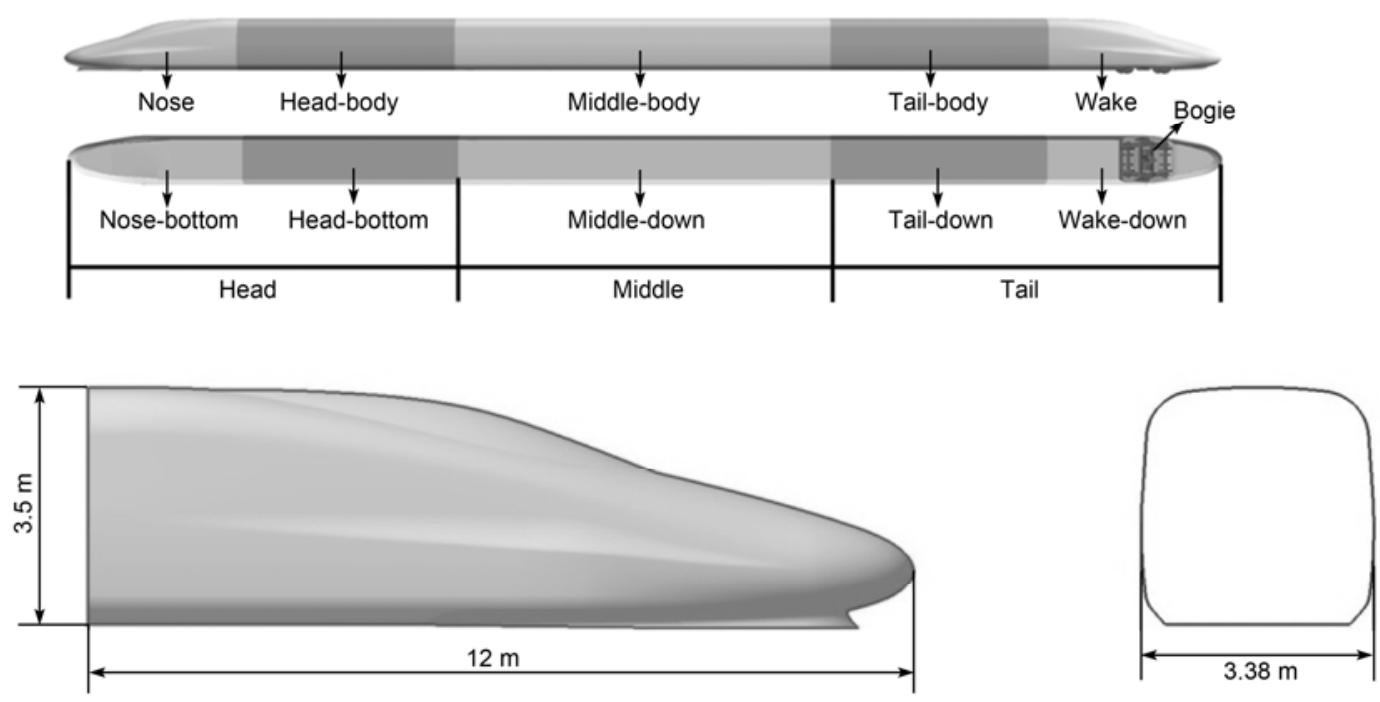

Figure 3 The simplified model of the CRH380A high-speed train.

based on the finite volume method are used to predict the aerodynamic drag. Roe's FDS scheme is used to calculate convective fluxes, and Lower-Upper Symmetric GaussSeidel (LU-SGS) is chosen for temporal discretization. The $k-\omega$ SST model is selected as the turbulence model. The standard wall functions are used near the wall so that the accuracy of the CFD results could be ensured with a limited amount of mesh.

Computational domains and boundary conditions: taking the length of the simplified train as the characteristic length $L$, then the length of inflow direction is $1 L$, the length of outflow direction is $2 L$, the width is $1 L$, the distance between the bottom of the train and the ground is $0.00235 \mathrm{~L}$ and the height is $0.5 \mathrm{~L}$, as shown in Figure 4. The flow velocity is $83.33 \mathrm{~m} \mathrm{~s}^{-1}$; the far-field pressure is $1 \mathrm{~atm}$, the temperature is $288 \mathrm{~K}$ and the reference area is the maximum cross-sectional area of the train. As a result of the compressibility calculation model, one-dimensional inviscid flow of the Riemann invariants is introduced as the far-field boundary conditions, which are also known as non-reflective boundary conditions. Inflow, outflow and the top boundaries are all set as far-field boundary conditions and the train body is non-slip solid wall boundary condition. The ground is treated as the moving wall so as to simulate the ground effect, and the moving speed is equal to the train speed.

\subsection{Grid-independent validation}

Mesh quality has a direct impact on the accuracy and stability of the calculation results. Thus, grid-independent validation is firstly performed in the present paper with different amounts of spatial mesh that combine prism mesh near the wall and hexahedral mesh so as to assess the influence of different spatial mesh on the calculation results. With the thickness of the first prism layer meeting the requirement of the wall function, three sets of mesh are used in the present paper, and the amount of the first set of the mesh is about 10.86 million, of which 6 prism layers are considered with a stretching ratio of 1.5 and the minimal length of the grid is about $12 \mathrm{~mm}$. For comparison, the second set of the mesh contains 9 prism layers with a stretching ratio of 1.2 and its minimal length of the grid is the same as the first set. The third set of the mesh contains 9 prism layers with a stretching ratio of 1.2 and the minimal length of the grid is $6 \mathrm{~mm}$. The amounts of the second and the third sets of mesh are 14.67 million and 21.04 million, respectively. The distribution of spatial grids is shown as Figure 5.

Table 1 shows the computational results of the three sets of mesh. As Table 1 shows, little influence on the results could be observed among the three sets of mesh. The variation of the predicted drags from the three sets of mesh is less
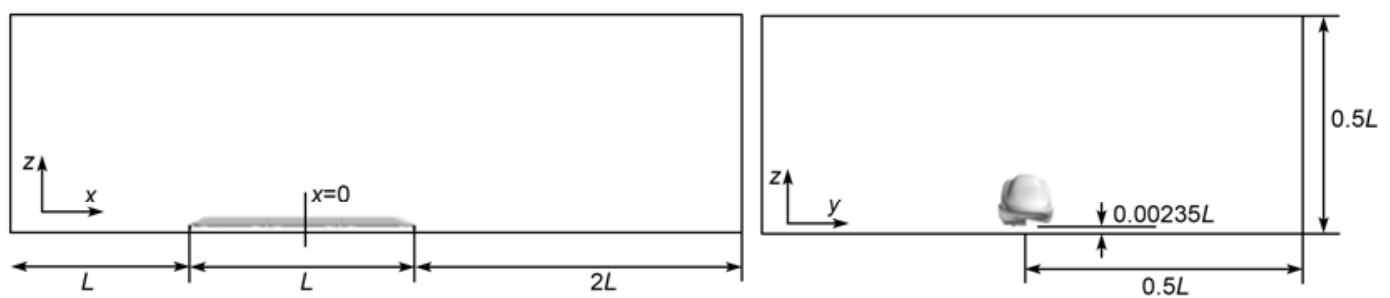

Figure 4 Computational domain of the flow field. 

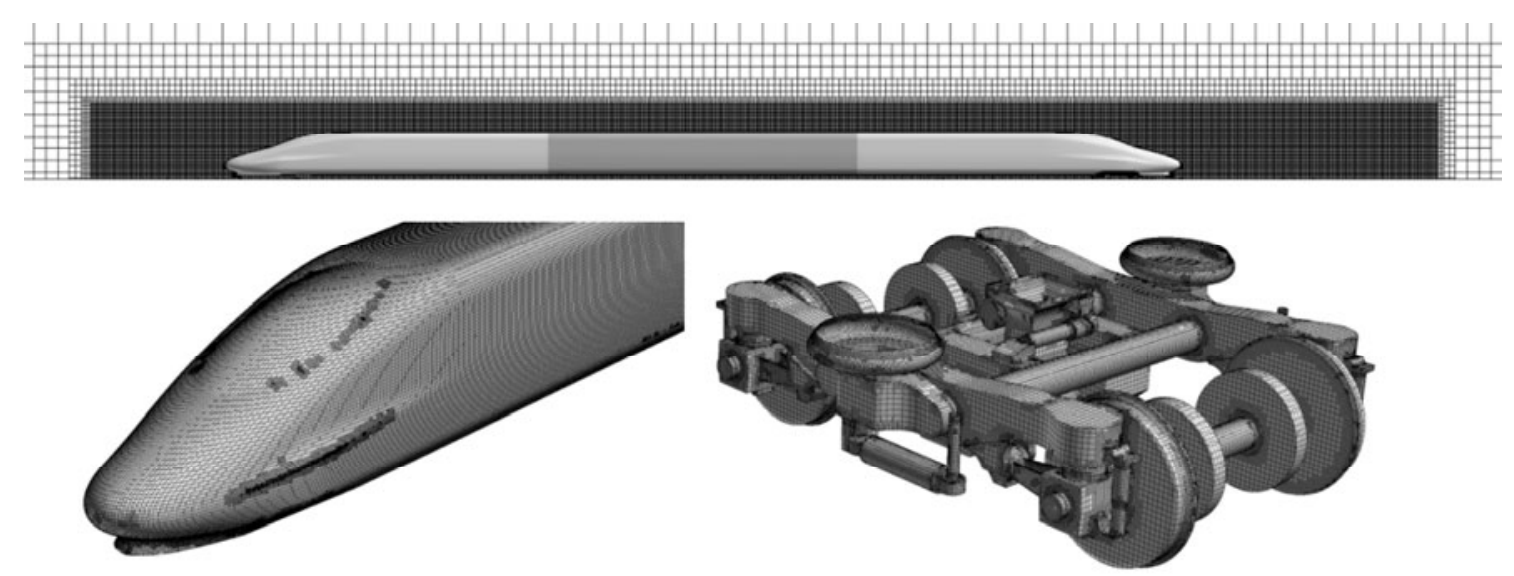

Figure 5 Distribution of spatial grids.

Table 1 Computational results of the three sets of mesh

\begin{tabular}{ccccc}
\hline $\begin{array}{c}\text { Mesh number } \\
\text { (million) }\end{array}$ & Total $C d$ & Tail $C l$ & Pressure drag & Shear drag \\
\hline 10.86 & 0.1971 & 0.0546 & 0.0660 & 0.1311 \\
14.67 & 0.1974 & 0.0560 & 0.0658 & 0.1316 \\
21.04 & 0.1975 & 0.0563 & 0.0669 & 0.1306 \\
\hline
\end{tabular}

than $1 \%$ while the variation of the lifts of the trailing car is less than 3\%. Moreover, the inviscid drag and the viscous drag from the three sets of the mesh are more or less the same. Therefore, it can be concluded that the mesh has less influence on the computational results. Considering the computational efficiency, the first set of mesh is utilized in the present paper.

In order to verify the reasonability of the simplified shape of Model-I, two additional kinds of computational models have been investigated for comparison. Model-II is the most simplified model which eliminates all the bogies and shields. On the contrary, Model-III takes all the bogies and shields into consideration. Table 2 shows the computational results of the three models. The predicted aerodynamic drags from the three models are all positive, varying apparently from each other. The lifts of the trailing car from Model-I and Model-III are both positive, yet the lift of trailing car of Model-II is negative, indicating that the last bogie beneath the streamline of the trailing car imposes a significant influence on the aerodynamic performance of the trailing car. Therefore, the simplification style of Model-I is much more reasonable.

Table 2 Computational results of the three models

\begin{tabular}{ccccc}
\hline Model type & Total $C d$ & Tail $C l$ & Pressure drag & Shear drag \\
\hline Model-I & 0.1971 & 0.0546 & 0.0660 & 0.1311 \\
Model-II & 0.1567 & -0.0237 & 0.0231 & 0.1336 \\
Model-III & 0.3224 & 0.0366 & 0.2146 & 0.1078 \\
\hline
\end{tabular}

\section{Multi-objective adaptive genetic algorithm}

As to Elitist Non-dominated Sorting Genetic Algorithm (NSGA-II), a large population $\mathrm{Rn}$ is combined with the parent population $\mathrm{Pn}$ and child population Qn, then the combined population is assigned to different parts based on the solutions of individual non-dominated relationship [21]. After that, all of the individuals are assigned a non-dominated rank equal to the non-dominated front label. Then the $N$ (number of initial population) individuals are selected from the beginning of the first non-dominated solutions of the combined population to construct a new population. In this paper, based on the concept of non-domination in NSAG-II, only the first non-dominated solutions of parent and child population are combined and recorded to get the first non-dominated solutions of new population. Thus, an adaptive non-dominated multi-objective genetic algorithm based on changing the crossover and mutation operators adaptively is proposed [22]. Besides, niche technology is used to ensure the diversity of the Pareto-optimal solutions and avoid converging to a local optimal solution. At present, the real coding and binary coding methods are usually used as the genetic algorithm encoding. The real coding method benefits from maintaining population diversity when performing the mutation operation, while binary encoding method can enhance the search capabilities of GA. As niche technology can ensure population diversity, in order to enhance the search capabilities of GA, binary encoding method with 22 bits is used both in the single objective genetic algorithm and multi-objective genetic algorithm.

The adaptive formulas [23] for the crossover probability and mutation probability during the evolution process are

$$
P_{\mathrm{c}}(\boldsymbol{x})=\left\{\begin{array}{l}
P_{\mathrm{c} 1}-\frac{\left(P_{\mathrm{c} 1}-P_{\mathrm{c} 2}\right)\left(f^{\prime}-f_{\text {avg }}\right)}{f_{\max }-f_{\text {avg }}}, f^{\prime} \geqslant f_{\text {avg }}, \\
P_{\mathrm{c} 1}, f^{\prime}<f_{\text {avg }},
\end{array}\right.
$$




$$
P_{\mathrm{m}}(\boldsymbol{x})=\left\{\begin{array}{l}
P_{\mathrm{m} 1}-\frac{\left(P_{\mathrm{m} 1}-P_{\mathrm{m} 2}\right)\left(f_{\mathrm{max}}-f\right)}{f_{\text {max }}-f_{\text {avg }}}, f \geqslant f_{\mathrm{avg}}, \\
P_{\mathrm{m} 1}, f<f_{\mathrm{avg}},
\end{array}\right.
$$

where $P_{\mathrm{c}}$ and $P_{\mathrm{m}}$ represent the crossover probability and mutation probability respectively, $f_{\max }$ is the maximal fitness, $f_{\text {avg }}$ the averaged fitness, $f^{\prime}$ the bigger fitness of the two individuals which need to cross over, $f$ the fitness of the individual that needs to mutate.

The fitness function of the individuals in the population which is based on the non-dominant rank is

$$
f i t_{i}(\boldsymbol{x})=\frac{1}{[1+\phi(\boldsymbol{x})+\psi(\boldsymbol{x})] \sum_{j}^{N} \operatorname{Sh}\left(d_{i j}\right)},
$$

where

$$
\phi(\boldsymbol{x})=(d-1) / D_{\text {rank }}
$$

which is the distance penalty function of the $d$-th individuals. $D_{\text {rank }}$ is the biggest class number in the local generation. The value of the distance penalty function in the same class keeps constant, which could ensure that the distance of the individuals in the same class keeps the same.

$$
\begin{gathered}
\psi(\boldsymbol{x})=\sum_{i=1}^{l} \alpha_{i}\left|\min \left\{0, g_{i}(\boldsymbol{x})\right\}\right|^{r}, \\
g_{i}(\boldsymbol{x}) \leqslant 0, i=1, \cdots, l .
\end{gathered}
$$

This is a constrained penalty function, and eq. (16) is the inequality expression of the constraint, where $l$ is the number of inequality constraints, $\alpha_{i}$ the coefficient of the penalty factor whose value is $1, r$ the scale of the penalty factor whose value is 2 . In the present paper only the volume constraint is considered.

$$
\operatorname{sh}\left(d_{i j}\right)=\left\{\begin{array}{l}
1-\frac{d_{i j}}{\sigma_{\text {share }}}, d_{i j} \leqslant \sigma_{\text {share }}, \\
0, d_{i j} \leqslant \sigma_{\text {share }} .
\end{array}\right.
$$

This is the sharing function used in the niche technology, where $d_{i j}$ is the distance between individual $i$ and individual $j, \sigma_{\text {share }}$ the niche radius, taking the form as

$$
\sigma_{\text {share }}=\frac{\sum_{i=1}^{N} \sum_{j=1}^{N} d_{i j}}{N \times N}, d_{i j}=\sqrt{\sum_{k=1}^{m}\left(x_{i, k}-x_{j, k}\right)^{2}},
$$

where $m$ is the number of the design variables and $x_{i, k}$ is the $k$-th normalized design variable of the individual $i$.

\section{Construction of Kriging model and predic- tion accuracy analysis}

\subsection{Sampling model}

In the present paper, a multi-objective optimization has been performed with the total drag of the whole train and the lift of the trailing car as the objectives. Meanwhile, a volume constraint has been adopted into the optimization which requires that the volume of optimal shape should be no less than $95 \%$ of the volume of the initial model. Under this constraint a Pareto set of the objectives could be obtained. In order to better parameterize the streamlined head, the normalization of the streamlined head along the axis of the initial train has been performed. When the CFD analysis was performed, the normalized length of the streamlined head was remapped to the real length. In the present paper 5 design variables have been proposed: the node drainage along the streamline $w_{1}$, the nose height $w_{2}$, the width of the streamline $w_{3}$, the slope of the cab window $w_{4}$ and the shift of the cowcatcher $w_{5}$. The design space of these variables is listed as

$$
\begin{aligned}
& -5 \leqslant w_{1} \leqslant 5, \quad-15 \leqslant w_{2} \leqslant 10, \quad-5 \leqslant w_{3} \leqslant 20, \\
& -10 \leqslant w_{4} \leqslant 4, \quad-60 \leqslant w_{5} \leqslant 10 .
\end{aligned}
$$

The basis for constructing a Kriging model is the sample information. A uniform distribution of sample points could provide the detailed information in the design space, making the model precisely capture the variation tendency of the objective. The Latin hypercube sampling method could ensure the sampling points to represent the whole part of the design space without taking the dimension of the problem into consideration. However, this method suffers from the unstable problem due to the randomness. In order to avoid this problem, the central Latin hypercube sampling method with minimax criteria based on the iterative local search algorithm has been utilized [24, 25].

Twenty one initial sampling points have been chosen for training the Kriging model, of which the first 20 points are chosen as training points while the last one is chosen as the test sampling point, just as Table 3 shows. It can be seen that compared to the total drag coefficient, the lift coefficient of the trailing car is more sensitive to the variation of the design variables, which requires more strictly the prediction accuracy of the Kriging model.

\subsection{Construction of Kriging model and accuracy anal- ysis}

Considering the huge computational cost in the aerodynamic optimization, especially in the shape optimization of high-speed trains, a sequential optimization method [14] based on minimizing the response surface criteria [13] has been adopted in the present paper so as to reduce the training points. A basic Kriging model could be firstly constructed from the initial 20 training points. Based on this model, the Pareto set could be obtained. Two typical points of the Pareto set could be chosen for CFD validation. If the optimization accuracy could not meet the requirement, these two points then would be added to the initial training points so as to build the Kriging model more accurately. If the 
Table 3 Initial training points and test points

\begin{tabular}{|c|c|c|c|c|c|c|c|c|}
\hline Type & Number & $w_{1}(\mathrm{~mm})$ & $w_{2}(\mathrm{~mm})$ & $w_{3}(\mathrm{~mm})$ & $w_{4}(\mathrm{~mm})$ & $w_{5}(\mathrm{~mm})$ & $C d$ & $\mathrm{Cl}$ \\
\hline \multirow{20}{*}{ Training points } & 1 & 0.0 & 0.0 & 0.0 & 0.0 & 0.0 & 0.1971 & 0.0546 \\
\hline & 2 & -2.75 & -5.625 & 11.875 & 0.85 & -23.25 & 0.2004 & 0.0668 \\
\hline & 3 & -1.25 & -9.375 & 4.375 & -8.95 & -44.25 & 0.1946 & 0.0563 \\
\hline & 4 & -4.75 & -10.625 & 9.375 & -2.65 & -9.25 & 0.1996 & 0.0600 \\
\hline & 5 & -4.25 & -11.875 & 0.625 & -0.55 & -37.25 & 0.1941 & 0.0548 \\
\hline & 6 & -2.25 & -0.625 & -1.875 & -3.35 & -26.75 & 0.1941 & 0.0519 \\
\hline & 7 & -0.75 & -1.875 & -0.625 & -1.25 & -51.25 & 0.1924 & 0.0524 \\
\hline & 8 & 0.75 & -8.125 & 15.625 & -4.05 & -33.75 & 0.1999 & 0.0714 \\
\hline & 9 & -3.25 & -14.375 & 16.875 & -9.65 & 1.25 & 0.2022 & 0.0756 \\
\hline & 10 & 3.75 & -4.375 & 6.875 & -8.25 & -54.75 & 0.1941 & 0.0575 \\
\hline & 11 & 4.75 & 0.625 & 18.125 & -4.75 & -40.75 & 0.2013 & 0.0709 \\
\hline & 12 & -0.25 & 4.375 & 13.125 & -1.95 & -47.75 & 0.1991 & 0.0611 \\
\hline & 13 & -3.75 & 3.125 & 1.875 & -7.55 & -16.25 & 0.1965 & 0.0543 \\
\hline & 14 & 2.75 & -6.875 & 5.625 & 2.95 & -30.25 & 0.1983 & 0.0599 \\
\hline & 15 & -1.75 & -3.125 & 3.125 & 3.65 & 8.25 & 0.1992 & 0.0572 \\
\hline & 16 & 1.75 & 6.875 & -3.125 & -6.85 & 4.75 & 0.1964 & 0.0521 \\
\hline & 17 & 1.25 & -13.125 & 10.625 & 2.25 & -5.75 & 0.2015 & 0.0643 \\
\hline & 18 & 4.25 & 5.625 & 8.125 & 0.15 & -19.75 & 0.2015 & 0.0618 \\
\hline & 19 & 2.25 & 8.125 & -4.375 & -6.15 & -12.75 & 0.1958 & 0.0515 \\
\hline & 20 & 0.25 & 9.375 & 14.375 & -5.45 & -58.25 & 0.1960 & 0.0597 \\
\hline Test points & 21 & 3.25 & 1.875 & 19.375 & 1.55 & -2.25 & 0.1970 & 0.0603 \\
\hline
\end{tabular}

optimization accuracy meets the design requirement, the Kriging model is finally constructed. The aerodynamic drag of the whole train hardly varies in the design space while the lift of the trailing car varies apparently. With the use of the same training points, the prediction accuracy of the Kriging model for the drag could limit its error within $1 \%$ while the accuracy for the lift of the trailing car only limits its error within $5 \%$.

Basically, the essence of training the Kriging model is to find the optimal value of $\theta_{k}$, which is to obtain the optimal solution of eq. (6). This could be done by maximum likelihood estimation [26] or schema search method [27]. However, both the methods suffer from specific problems. The former would suffer from falling into the local optimal solutions while the latter badly depends on the initial points. As a result, eq. (5) is chosen to be the objective and the optimal value of $\theta_{k}$ could be obtained through the adaptive GA approach based on 22-bit binary coding. Since each objective is corresponding to a variance $\sigma_{z}^{2}$, the optimization of eq. (6) belongs to a multi-objective, multi-variable optimization problem without constraints. In order to simplify the optimization yet still keep the prediction accuracy of the Kriging model, the multiple objectives are simply weighted to be a single objective. In the present paper, the two objectives are evenly weighted. As parameters for GA operation, the size of initial population is set to 500 . The probabilities of crossover are $P_{\mathrm{c} 1}=0.9, P_{\mathrm{c} 2}=0.6$ while the probabilities of mutation are set to $P_{\mathrm{m} 1}=0.1, P_{\mathrm{m} 2}=0.001$, respectively. The size of evolution generation is set to 1000. Figure 6 shows the convergence history of the fitness of the optimal solutions when first adding training points. After evolving 600 generations, the fitness converges to a constant value, indicating that the multi-objective optimization problem could be solved via a single-objective optimization problem.

Figure 7 shows the prediction error for the sampling points and points from the Pareto front. It can be seen that prediction of $\mathrm{Cl}$ from Kriging model is much worse than that of $C d$. As the training points increase, the prediction

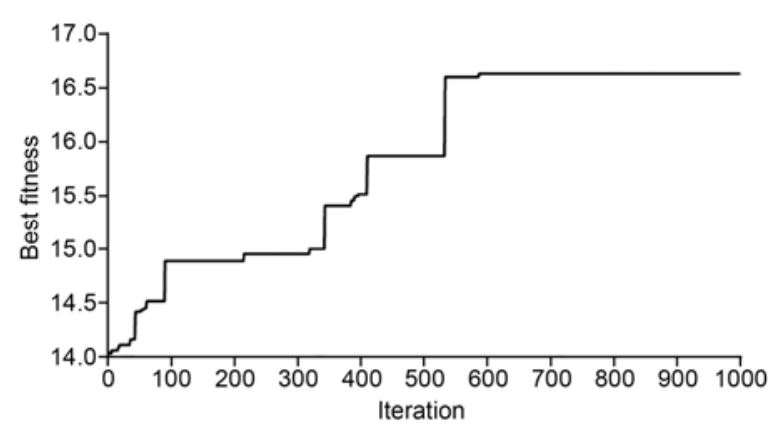

Figure 6 Convergence history of the best fitness. 

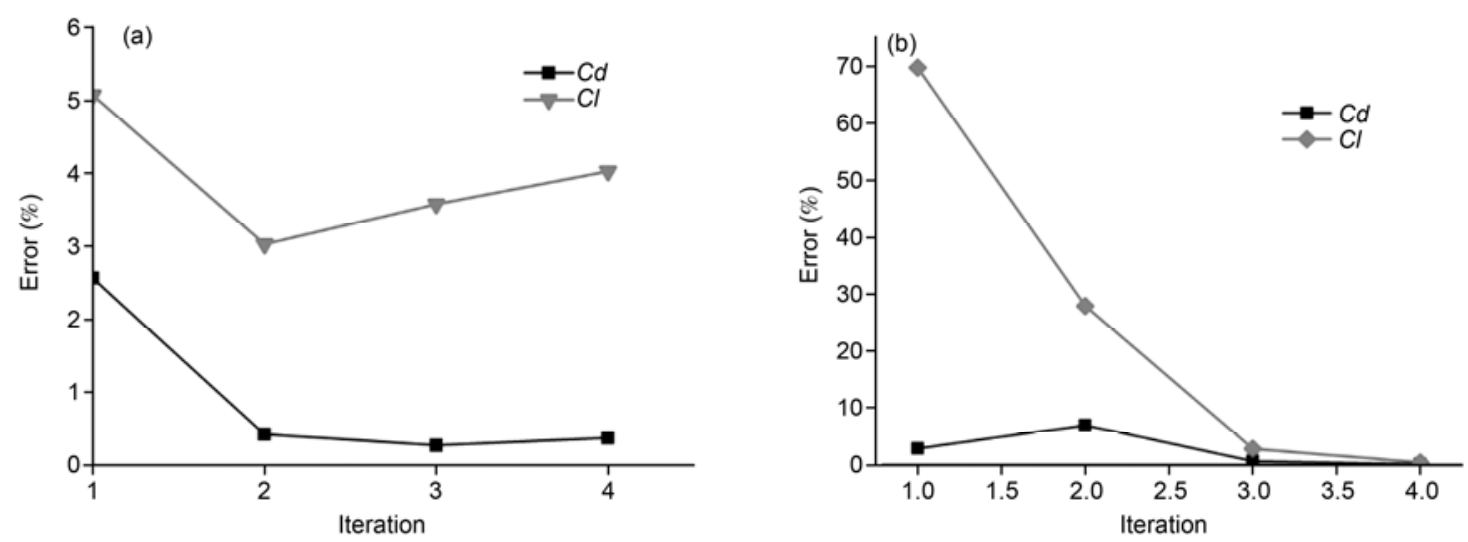

Figure 7 Prediction error of the sampling points and points from Pareto front. (a) Prediction accuracy of the sampling points; (b) prediction accuracy of the points from Pareto front.

accuracy of the Kriging model keeps unchanged in the design space. However, the prediction accuracy improves obviously in the Pareto front. When the first point is added, the prediction accuracy of the sampling points could meet the design requirement. However, huge error exists in the solutions of the Pareto front. After adding the second point, the prediction accuracy of the Kriging model has been improved greatly. After adding 4 points, the prediction accuracy of the sampling points and the Pareto front meets the engineering requirement.

\subsection{Optimization process}

Figure 8 shows the whole optimization process designed in the present paper. First, the Latin hypercube method is adopted to initialize the sampling points in the design space.
Then the CFD analysis is performed to obtain the values of the objectives corresponding to the sampling points. Thirdly, the Kriging model is constructed via GA technique based on these sampling points. After the Kriging model has been constructed, the multi-objective optimization based on self-adaptive GA approach could be performed and the Pareto set could be obtained. Two typical points of the Pareto set could be chosen for CFD validation. If the optimization accuracy could not meet the requirement, these two points then would be added to the initial training points so as to build the Kriging model more accurately. If the optimization accuracy meets the design requirement, the response surface based on the optimization objectives is finally constructed. In the end, the final Kriging model and multi-objective self-adaptive GA approach are both adopted to obtain the Pareto solutions in the design space.

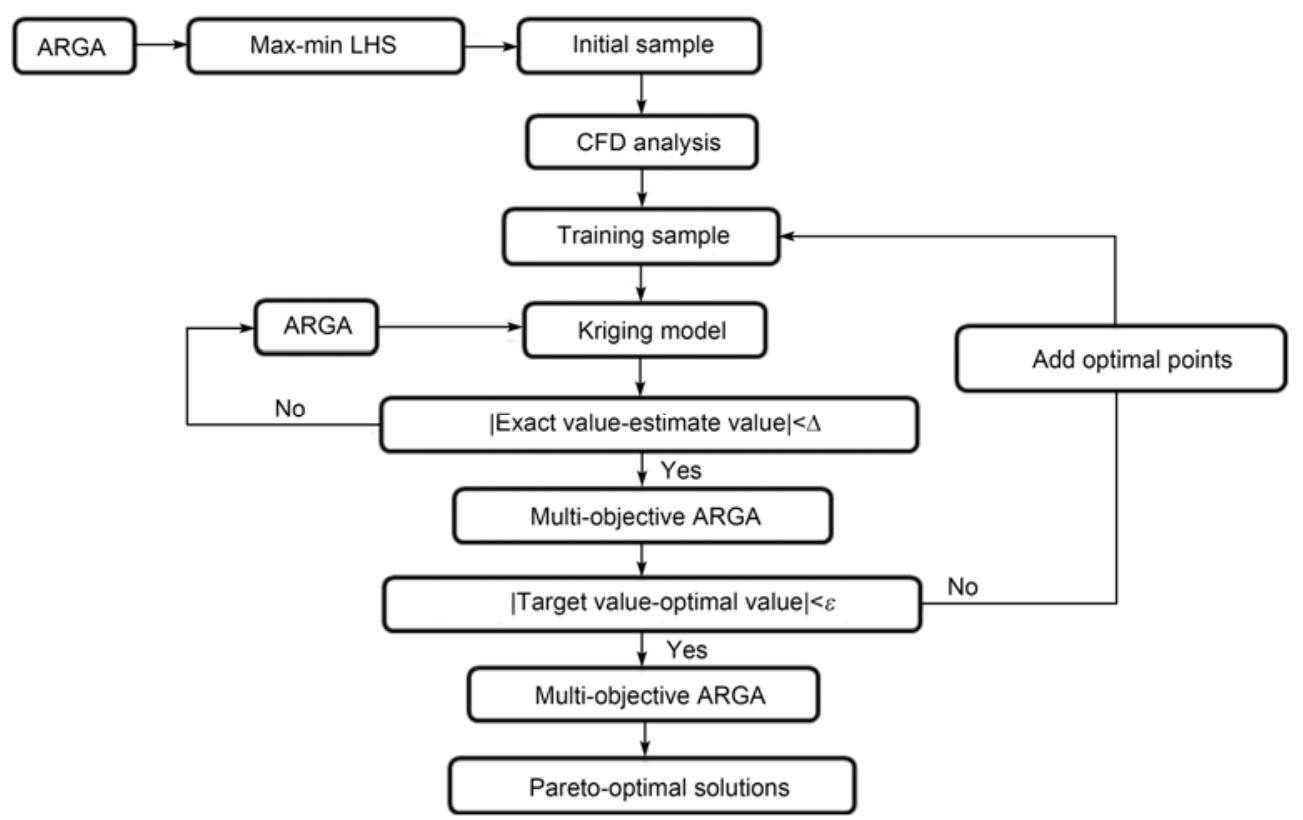

Figure 8 Schematic drawing of the optimization process. 


\section{Results and discussion}

\subsection{Comparative study between the initial shape and optimal shape}

The total aerodynamic drag coefficient and the lift of the trailing car are treated as the optimization objectives, and the adaptive genetic approach with a population number of 500 and 3000 generations has been performed in the optimization process. The roulette method is used as the selection operator, while the probabilities of crossover and mutation are set as: $P_{\mathrm{c} 1}=0.9, P_{\mathrm{c} 2}=0.6, P_{\mathrm{m} 1}=0.1, P_{\mathrm{m} 2}=0.001$. Figure 9 shows the Pareto front of the two objectives. It can be concluded that the optimal solutions of the objectives are both limited in a small zone, indicating that specific individual in the Pareto set shows no large differences, while all the individuals are better than the initial one. As a result, a specific individual is chosen in Figure 9 as an example, just as the circular spot shows.

Taking the circular spot in Figure 9 as an example, for the optimal shape, the middle point of the flow channel on the streamline has been lowered by $0.0149 \mathrm{~m}$, the height of the nose has been lowered by $0.024 \mathrm{~m}$, the width of the streamline has been narrowed with a maximal value of $0.1165 \mathrm{~m}$, the angle of the window on top of the streamline has decreased by $1.99^{\circ}$, and the cowcatcher has been put backward with a value of $0.569 \mathrm{~m}$. Meanwhile, the volume of the streamline changes from $89.7178 \mathrm{~m}^{3}$ to $87.7824 \mathrm{~m}^{3}$, which meets the constraints in the optimization. The aerodynamic force coefficients of the optimal and original shapes are given in Table 4, indicating that the results from the Kriging model and CFD are almost the same. After optimization, the total drag coefficient has been reduced by $3.2 \%$, and the lift of the trailing car has been reduced by
$8.24 \%$. The viscous force of the total train is almost the same, while the inviscid force has been reduced by $10.02 \%$.

Figure 10 shows the pressure contour around the streamline of the optimal shape and original shape. It can be observed that an apparent high pressure zone exists around the nose and cowcatcher, and a slightly lower high pressure zone exists around the connection zone between the nose

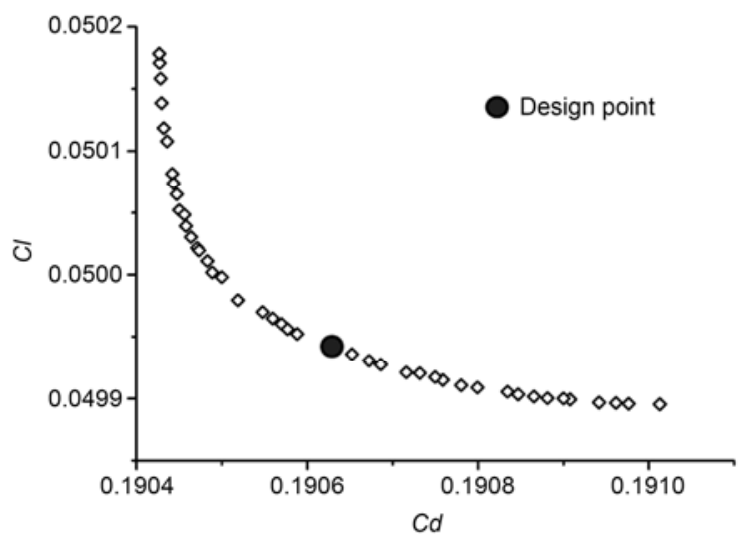

Figure 9 Pareto front based on the drag of the whole train and the lift of the trailing car.

Table 4 Aerodynamic force coefficients of the optimal and original shapes

\begin{tabular}{ccccc}
\hline Model type & Total $C d$ & Tail $C l$ & Pressure drag & Shear drag \\
\hline Original shape & 0.1971 & 0.0546 & 0.0659 & 0.1312 \\
Optimal shape & 0.1908 & 0.0501 & 0.0593 & 0.1315 \\
Reduction & $3.20 \%$ & $8.24 \%$ & $10.02 \%$ & $-0.23 \%$ \\
Kriging model & 0.1906 & 0.0499 & - & - \\
\hline
\end{tabular}

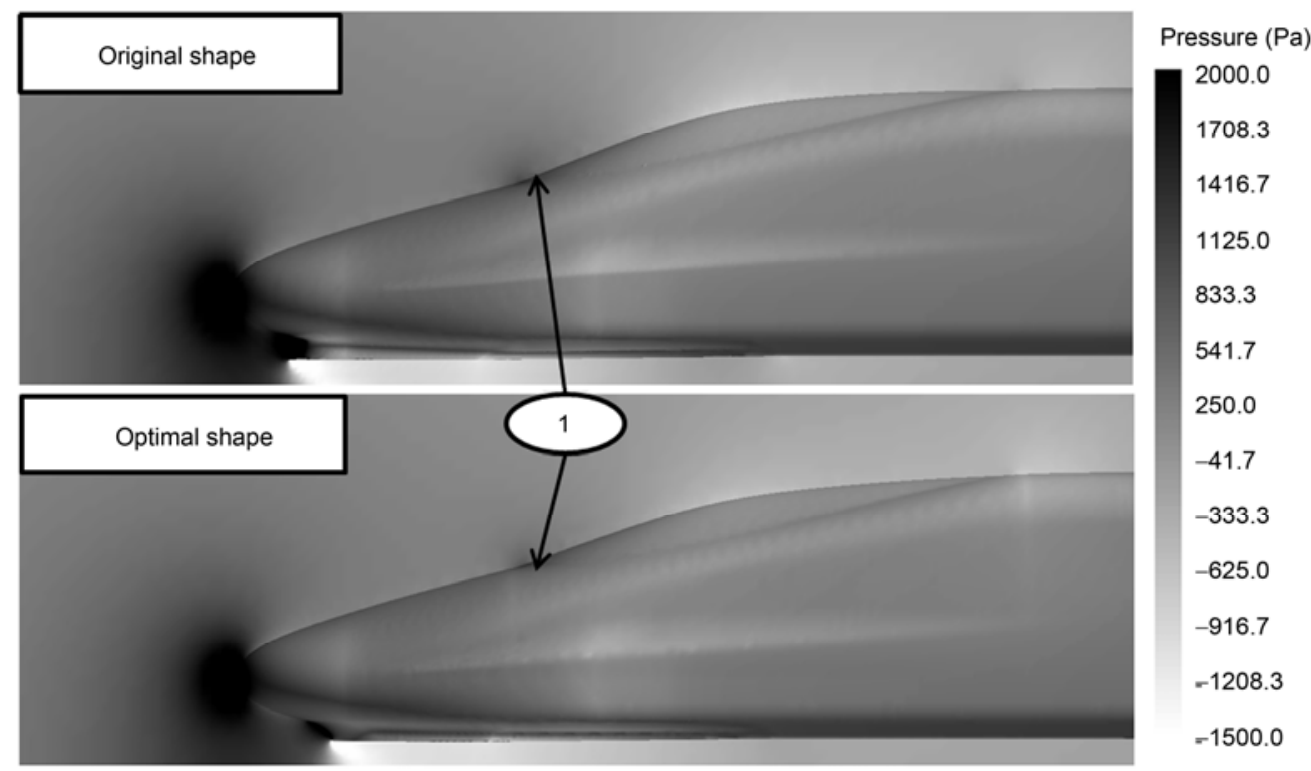

Figure 10 Pressure contour around the streamline of the optimal shape and original shapes. 
and the window. Meanwhile, an apparent low pressure zone exists beneath the cowcatcher. After the optimization, the cowcatcher has been moved backward, which slows the accelerating process of the passing flow. As a result, the high pressure zone here has been reduced. Meanwhile, as the height of the nose changes with the slope of the window, the high pressure zone around the connection area also has been alleviated. Therefore, the inviscid force of the leading car has been reduced.

Figure 11 shows that the pressure coefficient $(C p)$ on the upper surface keeps almost the same between the original shape and optimal shape, only with small differences at the connection part between the nose and the cab window. Meanwhile, because of the shift of the cowcatcher, the pressure coefficient on the lower surface changes a lot. The pressure coefficient along the surface of the trailing streamline has also been drawn in Figure 11 for comparison. It can be seen that the pressure coefficient gets little difference between the original and optimal shapes, which could be attributed to thicker boundary layer there.
In order to better understand the aerodynamic performance of the optimal streamline and the influence on other parts due to the change of the streamline, the aerodynamic drag coefficient and lift coefficient of different parts of the original train and the optimal train are listed in Table 5. It can be seen that the inviscid drag mainly exists around the nose of the leading and trailing streamline. The inviscid drag of the trailing nose is about $55 \%$ of the total inviscid drag. The underbody parts of the train experience negative lifts, which would drag down the train body. The lifts of the other parts of the train are all positive, which would exert an outward force on the train body. The inviscid drag of the leading and trailing streamline has been reduced a lot, with a value of 0.003 , while the drag of other parts keeps unchanged. The trailing streamline experiences an evident reduction on the lift, while the lift of the trailing car becomes slightly higher and the negative lift of the bottom of the trailing car decreases. Maximal change of the lift happens on the trailing streamline, which is about 0.0057 , while the lift of the bottom surface of the trailing streamline and
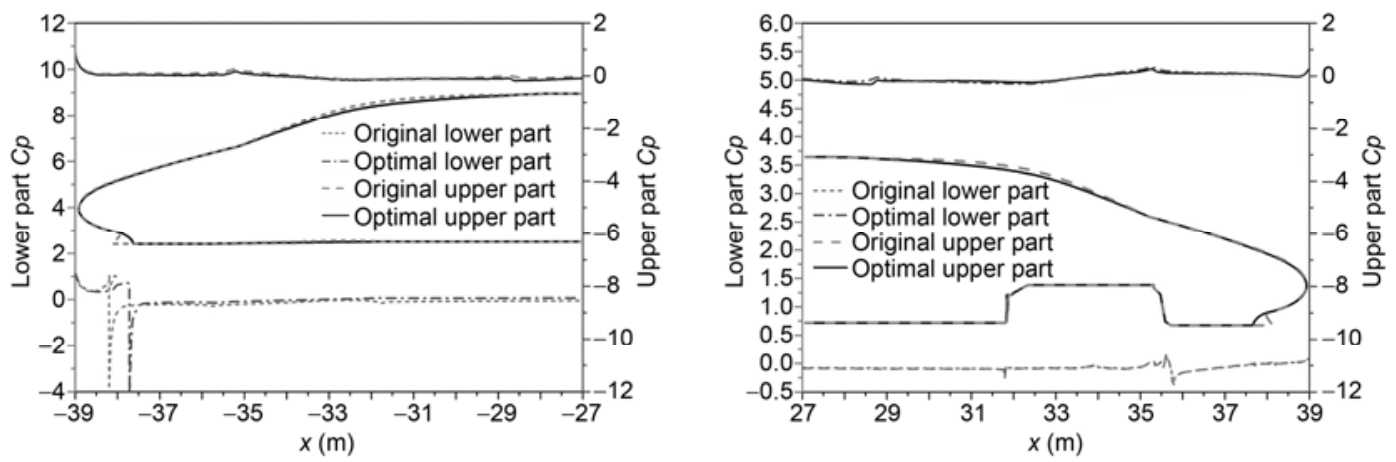

Figure 11 Pressure coefficient along the surface of the streamline.

Table 5 Drag coefficient and lift coefficient of different parts of the original train and the optimal train

\begin{tabular}{|c|c|c|c|c|c|c|}
\hline \multirow{2}{*}{ Train part } & \multicolumn{2}{|c|}{ Original shape } & \multicolumn{2}{|c|}{ Optimal shape } & \multirow{2}{*}{$\begin{array}{c}\text { Original shape } \\
\text { tail } \mathrm{Cl}\end{array}$} & \multirow{2}{*}{$\begin{array}{c}\text { Optimal shape } \\
\text { tail } \mathrm{Cl}\end{array}$} \\
\hline & pressure & shear & pressure & shear & & \\
\hline Nose & 0.0060 & 0.0156 & 0.0030 & 0.0156 & - & - \\
\hline Nose-down & 0.0020 & 0.0045 & 0.0017 & 0.0047 & - & - \\
\hline Head-body & 0.0000 & 0.0218 & 0.0000 & 0.022 & - & - \\
\hline Head-down & 0.0000 & 0.0064 & 0.0000 & 0.0064 & - & - \\
\hline Middle-body & 0.0000 & 0.0346 & 0.0000 & 0.0348 & - & - \\
\hline Middle-down & 0.0000 & 0.0099 & 0.0000 & 0.0097 & - & - \\
\hline Tail-body & 0.0000 & 0.0195 & 0.0000 & 0.0196 & 0.0800 & 0.0835 \\
\hline Tail-down & 0.0000 & 0.0052 & 0.0000 & 0.0052 & -0.0783 & -0.0803 \\
\hline Wake & 0.0366 & 0.0112 & 0.0336 & 0.011 & 0.1820 & 0.1763 \\
\hline Wake-down & 0.0115 & 0.0019 & 0.0111 & 0.0019 & -0.1179 & -0.1179 \\
\hline Total & 0.0659 & 0.1312 & 0.0593 & 0.1315 & 0.0546 & 0.0501 \\
\hline
\end{tabular}


the bogies keeps unchanged.

\subsection{Comparison of the real train model}

The above optimization of the streamline shape is based on a simplified train, so that the cost of mesh generation and CFD analysis could be greatly reduced. A proper simplification on the CRH380A has been performed. Only the bogies beneath the streamlined head have been reserved while other ancillary components are all neglected. However, these components impose an intense effect on the aerodynamic performance of the whole train. In order to take the effect of the ancillary components into consideration and further study the aerodynamic performance of the optimal train, a comparative study between the original prototype CRH380A and complicated optimal train has been performed. The real models with all the ancillary components are shown in Figure 12 .

The computational approach of the complicated models is the same as the simplified models. The hybrid mesh configuration has been adopted with the prism grids along the surface of the train and Cartesian grids in the outer of the prism grids. 6 layers of prisms have been generated with the initial height of $0.45 \mathrm{~mm}$ and a stretching ratio of 1.2. In order to obtain a finer flow field, the zones around the ancillary components are locally densified. The total amount of the mesh is about 22.3 million.

The distribution of aerodynamic forces of the CRH380A prototype and the optimal train has been shown in Table 6 . It could be obviously seen that the inviscid drag of the CRH380A prototype is greatly larger than the simplified one and the viscous drag is a little smaller. The inviscid drag of the first bogie beneath the leading streamline is slightly bigger which is a result of the impact of the incoming flow with the high speed. Due to the existence of a bogie cover under the streamline, the inviscid drag of the prototype CRH380A is greater than the simplified model. Large vortices are generated at the inter-spacing between adjacent cars which would produce negative drag on Shield- 1 and Shield-3 on the leeward side and produce positive drag on Shiled-2 and Shield-4 on the windward side. However, the algebraic sum of four shields remain a small value, indicating that the inter-spacing imposes a great effect on the aerodynamic force distribution of each car while having little influence on the total aerodynamic force. The lift of the trailing car arises from the pull force on the surface. However, due to negative lift by the ancillary components, the total lift of the trailing car of the prototype is a little smaller than the simplified one. The aerodynamic forces of the ancillary components keep basically unchanged before and after the optimization. With the same simplified model, the drag of the leading and trailing noses of the complicated model gets reduced greatly, and also the lift of the trailing streamline. Compared to the prototype CRH380A, the total drag of the optimal train has been reduced by $2.26 \%$, in which the inviscid drag has been reduced by $3.78 \%$, and the viscous drag increases by $0.74 \%$. The lift of the trailing car has been reduced by $19.67 \%$. Compared to the simplified model, the great change is mainly caused by the ancillary components (such as the bogies and the inter-spacing of the the train), indicating that the lift of the trailing car is very sensitive to the ancillary components and imposes a great challenge to the simplification of the train. Although the variation values of the simplified model and the prototype model are not the same, they both share the same tendency and could illustrate the effect on the aerodynamic force due to the change of the streamlined head. As a result, it is reasonable to utilize a simplified model to perform the optimization, just as the approach used in the present paper, which greatly reduces the cost of CFD analysis and improves the optimization efficiency.

\subsection{Comparison of aerodynamic performance in cross wind conditions}

Severe cross wind conditions are frequently met in reality, and the aerodynamic performance would deteriorate fiercely compared to conditions without cross wind, which would impose a threat on the running safety. In order to study the robustness of the optimal shape, the cross wind case has been investigated between the prototype CRH380A and the optimal train.

In the cross wind study, the running speed is set to be $300 \mathrm{~km} \mathrm{~h}^{-1}$, and the crosswind velocity is $15 \mathrm{~m} \mathrm{~s}^{-1}$ with an angle of $90^{\circ}$. The CFD algorithm and mesh configuration are both the same with the calculation in condition without cross wind, except that the leeward side of the train has been densified so as to precisely capture the vortex structures there. The total amount of the grids is about 26.2 million.

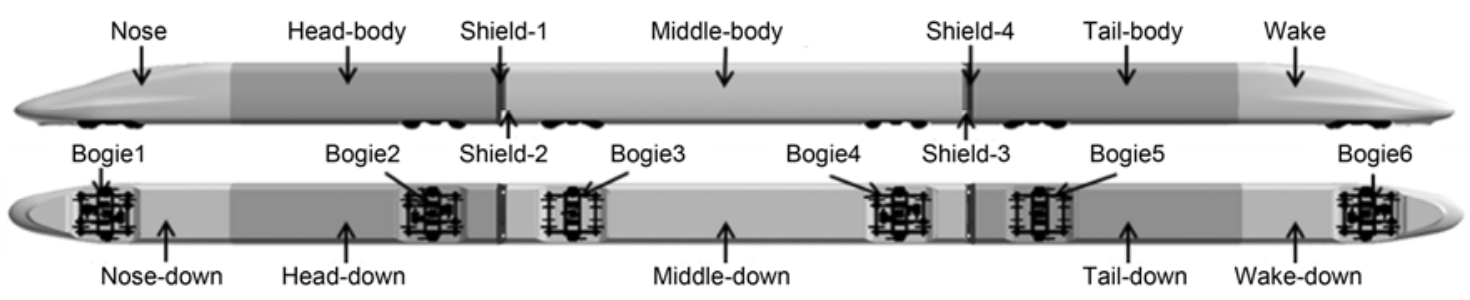

Figure 12 Practical CRH380A model with all the ancillary components. 
Table 6 Distribution of aerodynamic forces of the CRH380A prototype and the optimal train

\begin{tabular}{|c|c|c|c|c|c|c|}
\hline \multirow{2}{*}{ Train part } & \multicolumn{2}{|c|}{ Original shape } & \multicolumn{2}{|c|}{ Optimal shape } & \multirow{2}{*}{$\frac{\text { Original shape }}{\text { tail } \mathrm{Cl}}$} & \multirow{2}{*}{$\frac{\text { Optimal shape }}{\text { tail } C l}$} \\
\hline & pressure & shear & pressure & shear & & \\
\hline Nose & 0.0147 & 0.0145 & 0.0115 & 0.0145 & - & - \\
\hline Nose-down & 0.0353 & 0.0023 & 0.0308 & 0.0023 & - & - \\
\hline Head-body & 0.0000 & 0.0207 & 0.0000 & 0.0209 & - & - \\
\hline Head-down & 0.0102 & 0.0026 & 0.0097 & 0.0025 & - & - \\
\hline Bogie1 & 0.0322 & 0.0011 & 0.0331 & 0.0012 & - & - \\
\hline Bogie2 & 0.0075 & 0.0004 & 0.0081 & 0.0004 & - & - \\
\hline Shield-1 & -0.0332 & 0.0001 & -0.0343 & 0.0001 & - & - \\
\hline Middle-body & 0.0000 & 0.0320 & 0.0000 & 0.0325 & - & - \\
\hline Middle-down & 0.0113 & 0.0022 & 0.0122 & 0.0023 & - & - \\
\hline Bogie3 & 0.0087 & 0.0004 & 0.0091 & 0.0004 & - & - \\
\hline Bogie4 & 0.0053 & 0.0002 & 0.0043 & 0.0002 & - & - \\
\hline Shield-2 & 0.0485 & 0.0002 & 0.0496 & 0.0002 & - & - \\
\hline Shield-3 & -0.0279 & 0.0001 & -0.0287 & 0.0001 & - & - \\
\hline Tail-body & 0.0000 & 0.0180 & 0.0000 & 0.0182 & 0.0833 & 0.0832 \\
\hline Tail-down & 0.0042 & 0.0011 & 0.0057 & 0.0011 & -0.1194 & -0.1187 \\
\hline Wake & 0.0433 & 0.0107 & 0.0391 & 0.0106 & 0.1896 & 0.1813 \\
\hline Wake-down & 0.0081 & 0.0008 & 0.0046 & 0.0007 & -0.0899 & -0.0890 \\
\hline Bogie5 & 0.0042 & 0.0003 & 0.0047 & 0.0003 & -0.0061 & -0.0059 \\
\hline Bogie6 & 0.0043 & 0.0002 & 0.0035 & 0.0002 & -0.0062 & -0.0061 \\
\hline Shield-4 & 0.0423 & 0.0002 & 0.0432 & 0.0002 & -0.0147 & -0.0154 \\
\hline Total & 0.2143 & 0.1081 & 0.2062 & 0.1089 & 0.0366 & 0.0294 \\
\hline
\end{tabular}

Table 7 shows the aerodynamic force distribution of different components of the prototype CRH380A and the optimal train in cross wind condition. It can be observed that the aerodynamic drag of the whole train and the lift of the trailing car have increased greatly compared to those in condition without cross wind. A negative drag exists at the nose of the leading nose of the streamline while the drag of the whole leading car is positive. The aerodynamic forces of the leading and trailing streamline vary apparently after the optimization, while the aerodynamic forces of the other components keep unchanged. Compared to the prototype CRH380A, in cross wind conditions, the total drag of the optimal train has been reduced by $2.24 \%$, in which the inviscid force has been reduced by $3.02 \%$ while the viscous force keeps the same. The lift of the trailing car has been reduced by $10.27 \%$. The results reveal that the optimal shape shows excellent aerodynamic performance even in cross wind conditions.

\subsection{Sensitivity analysis}

Reasonable design variables greatly affect the optimization results. In this section further discussion of the design variables will be performed. For the already built Kriging model, the value of $\theta_{k}$ means the sensitivity to the optimization objective, so that it could be used to study the influence of the design variables on the optimization objectives [13]. Figure 13 shows the normalized value of $\theta_{k}$ of each design variable. It can be seen that these five values differ greatly compared to each other. Due to the narrow design space of the flow channel of the nose and the slope of the cab window, small variations would hardly disturb the initial flow field. As a result, the values of $\theta_{1}$ and $\theta_{4}$ corresponding to $w_{1}$ and $w_{4}$ are very small, indicating that the original design of the cab window and the flow channel along the streamline is very reasonable. Bigger values of $\theta_{3}$ and $\theta_{5}$ could be observed, which corresponds to the width of the train body and the shift of the cowcatcher, respectively. The windward area of the streamline is mainly controlled by the width of the train body, which will dominate the inviscid drag of the leading car. The design of the cowcatcher could affect not only the aerodynamic drag of the train, but also the intensity of the detachment of trailing vortices, and further the lift of the trailing car. The design variables utilized in the present paper all impose certain influence on the objectives, and could illustrate the key characteristics of the streamlined head, indicating that these design variables are chosen reasonably. 
Table 7 Force distribution of different components of the prototype CRH380A and the optimal train in cross wind condition

\begin{tabular}{|c|c|c|c|c|c|c|}
\hline \multirow{2}{*}{ Train part } & \multicolumn{2}{|c|}{ Original shape } & \multicolumn{2}{|c|}{ Optimal shape } & \multirow{2}{*}{$\frac{\text { Original shape }}{\text { tail } \mathrm{Cl}}$} & \multirow{2}{*}{$\frac{\text { Optimal shape }}{\text { tail } \mathrm{Cl}}$} \\
\hline & pressure & shear & pressure & shear & & \\
\hline Nose & -0.0342 & 0.0154 & -0.0372 & 0.0153 & - & - \\
\hline Nose-down & 0.0287 & 0.0021 & 0.0299 & 0.0020 & - & - \\
\hline Head-body & 0.0000 & 0.0230 & 0.0000 & 0.0226 & - & - \\
\hline Head-down & 0.0237 & 0.0039 & 0.0234 & 0.0039 & - & - \\
\hline Bogie1 & 0.0202 & 0.0008 & 0.0198 & 0.0008 & - & - \\
\hline Bogie2 & 0.0131 & 0.0007 & 0.0124 & 0.0007 & - & - \\
\hline Shield-1 & -0.0370 & 0.0000 & -0.0370 & 0.0000 & - & - \\
\hline Middle-body & 0.0001 & 0.0382 & 0.0001 & 0.0383 & - & - \\
\hline Middle-down & 0.0383 & -0.0060 & 0.0389 & 0.0059 & - & - \\
\hline Bogie3 & 0.0114 & 0.0006 & 0.0107 & 0.0006 & - & - \\
\hline Bogie4 & 0.0159 & 0.0008 & 0.0146 & 0.0008 & - & - \\
\hline Shield-2 & 0.0528 & 0.0002 & 0.0526 & 0.0002 & - & - \\
\hline Shield-3 & -0.0317 & 0.0001 & -0.0320 & 0.0001 & - & - \\
\hline Tail-body & 0.0000 & 0.0212 & 0.0000 & 0.0215 & 0.3389 & 0.3391 \\
\hline Tail-down & 0.0194 & 0.0039 & 0.0179 & 0.0037 & -0.1663 & -0.1701 \\
\hline Wake & 0.0784 & 0.0128 & 0.0763 & 0.0129 & 0.2962 & 0.2741 \\
\hline Wake-down & 0.0175 & 0.0026 & 0.0158 & 0.0024 & -0.1461 & -0.1500 \\
\hline Bogie5 & 0.0129 & 0.0007 & 0.0127 & 0.0007 & -0.0126 & -0.0116 \\
\hline Bogie6 & 0.0206 & 0.0011 & 0.0213 & 0.0010 & -0.0209 & -0.0206 \\
\hline Shield-4 & 0.0479 & 0.0002 & 0.0488 & 0.0002 & -0.0048 & -0.0057 \\
\hline Total & 0.2980 & 0.1343 & 0.2890 & 0.1336 & 0.2844 & 0.2552 \\
\hline
\end{tabular}

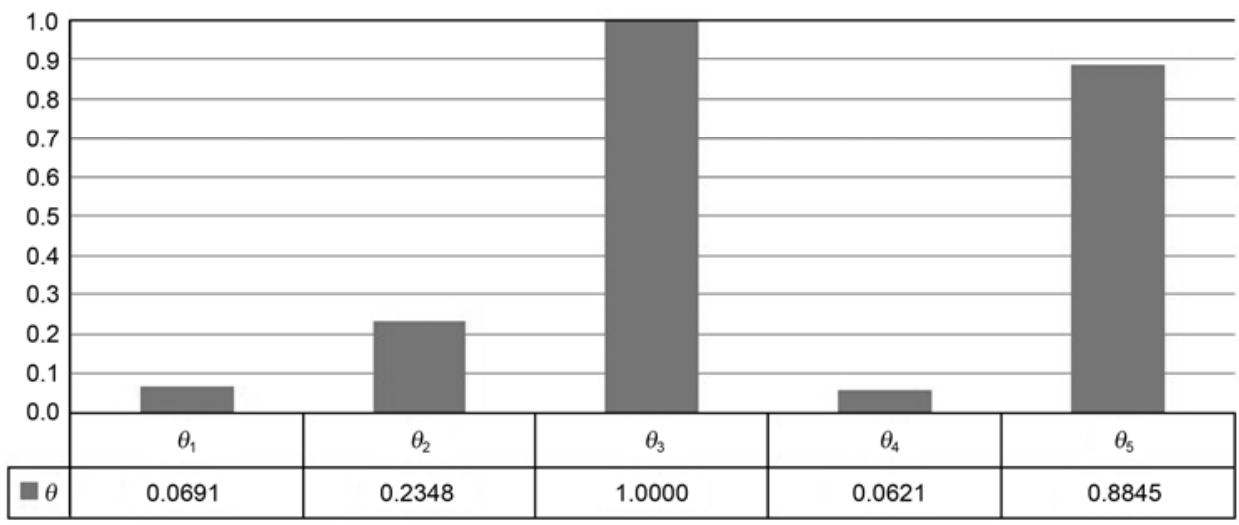

Figure 13 Normalized value of $\theta_{k}$ of each design variable.

\section{Conclusions}

Based on the simplified CRH380A model with three carriages, a multi-objective aerodynamic optimization has been performed. The volume of the streamlined head has been chosen as the constraint. Several conclusions have been obtained.

(1) A multi-objective aerodynamic shape optimization approach has been proposed in the present paper, which is based on the adaptive non-inferior classification genetic algorithm. This approach owns high optimization efficiency, which could be utilized in practical engineering problems, shedding light on the design of large scale, multi-variable, multi-objective aerodynamic shape optimization.

(2) With only a small amount of training points, the Kriging model based on the adaptive GA and minimizing the response surface criteria could be constructed, which could meet the accuracy requirement in engineering prob- 
lems. This model could be perfectly utilized in multi-objective optimization problems and improve the optimization efficiency.

(3) For the simplified CRH380A model, the total drag of the whole train has been reduced by $3.2 \%$, in which the inviscid drag has been reduced by $10.02 \%$ while the viscous drag keeps almost unchanged. The lift of the trailing car has been reduced by $8.24 \%$. Compared to the initial model, the volume of the streamline of the optimal train has been reduced by $2.16 \%$. For the prototype CRH380A model, the total drag of the whole train has been reduced by $2.26 \%$ and the lift of the trailing car has been reduced by $19.27 \%$. The difference of the aerodynamic performance is mainly due to the change of the streamline. Although the results from the simplified model and the prototype model get some differences, they both share the same tendency for the initial model and the optimal model, indicating that the simplified model could be utilized for optimization.

(4) Ancillary components could be a key factor that dominates the aerodynamic performance of the train. The inter-coach spacing could change the aerodynamic distribution on each carriage. However, it has little influence on the aerodynamic performance of the whole train. The lift of the trailing car is mainly caused by the pull on the surface of the train. The ancillary components experience a negative lift while the trailing nose experiences a positive lift.

(5) The optimal shape shows excellent aerodynamic performance in cross wind conditions. Compared to the initial model, the aerodynamic drag has been reduced by $2.24 \%$, in which the inviscid drag has been reduced by $2.02 \%$ while the viscous drag remains the same. The lift of the trailing car has been reduced by $10.27 \%$. Results reveal that the optimization of the streamline owns high robustness.

This work was supported by the Major State Basic Research Development Program of China ("973" Program) (Grant No. 2011CB711100) and National Key Technology R\&D Program (Grant No. 2009BAQG12A03).

1 Raghuathan S, Kim H D, Setoguchi T. Aerodynamics of high-speed railway train. Progr Aerosp Sci, 2002, 8(6): 469-514

2 Baker C. The flow around high speed trains. J Wind Eng Indus Aerodyn, 2010, 98(6-7): 277-298

3 Tian H Q. Formation mechanism of aerodynamic drag of high-speed train and some reduction measures. J Cent South Univ Technol, 2009, 16: $166-171$

4 Tian H Q. Train Aerodynamics. Beijing: China Railway Publishing House, 2007. 160-161
5 Ku Y C, Kwak M H, Park H I, et al. Multi-objective optimization of high-speed train nose shape using the vehicle modeling function. In: 48th AIAA Aerospace Sciences Meeting, 2010, Orlando, USA

6 Sun Z X, Song J J, An Y R. Optimization of the head shape of the CRH3 high speed train. Sci China Tech Sci, 2010, 12: 3356-3364

$7 \mathrm{Ku} \mathrm{Y} \mathrm{C,} \mathrm{Rho} \mathrm{J} \mathrm{H,} \mathrm{Su-Hwan,} \mathrm{et} \mathrm{al.} \mathrm{Optimal} \mathrm{cross-sectional} \mathrm{area} \mathrm{dis-}$ tribution of a high-speed train nose to minimize the tunnel micropressure wave. Struct Multidisc Optim, 2010, 42: 965-976

8 Lee J S, Kim J H. Approximate optimization of high-speed train nose shape for reducing micropressure wave. Indust Appl, 2008, 35: 79-87

9 Kwon H B, Jang K H, Kim Y S, et al.. Nose shape optimization of high-speed train for minimization of tunnel sonic boom. Jpn Soc Mech Eng, 2001, 4: 890-899

10 Lida M, Matsumura T, Nakatani K, et al. Effective nose shape for reducing tunnel sonic boom. QR of RTRI, 1997, 38(4): 206-211

11 Noel C. The origins of Kriging. Math Geol, 1990, 22(3): 239-252

12 Donald R J, Matthias S, William J W. Efficient global optimization of expensive black-box functions. J Global Optim, 1998, 13: 455-492

13 Donald R J. A taxonomy of global optimization methods based on response surfaces. J Global Optim, 2001, 21: 345-383

14 Gao Y H, Wang X C. An effective warpage optimization method in injection molding based on kriging model. Int J Adv Manuf Technol, 2008, 37(9): 953-960

15 Koehler J R, Owen A B. Computer experiments, in Ghosh S, Rao C R, eds. Handbook of Statistics. New York: Elsevier Science, 1996. 261-308

16 Welch W J, Yu T K, Kang S M, et al.. Computer experiments for quality control by parameter design. J Qual Technol, 1990, 22(1): $15-22$

17 Jamshid A S. Aerodynamic shape optimization based on free-form deformation. AIAA 2004-4630, 2004

18 Kulfan B M, Bussoletti J E. Fundamental parametric geometry representations for aircraft component shapes. 11th AIAA/ISSMO Multidisciplinary Analysis and Optimization Conference, Portsmouth, VA, 2006. 1-42

19 Rho J H, Ku Y C, Yun S H, et al. Development of vehicle modeling function for 3-dimensional shape optimization. J Mech Design, 2009, 131(12): 121004-1-121004-10

20 Blazek J. Computational Fluid Dynamics Principles and Applications, Oxford: Elsevier Ltd, 2005. 16-17

21 Pratab A, Deb K. A fast elitist non-dominated sorting genetic algorithm for multi-objective optimization: NSGA-II. Kan-GAL report 200001, Indian Institute of Technology, Kanpur, India, 2000

22 Yang G W, Chen D W, Cui K. Response surface technique for static aeroelastic optimization on a high-aspect-ratio wing. J Aircr, 2009, 46(4): 1444-1450

23 Srinivas M, Patnaik LM. Adaptive probalilities of crossover and mutations in GAs. IEEE T SMC, 1994, 24(4): 656-667

24 Roshan V J. Orthogonal-maximin Latin hypercubes designs. Stat Sin, 2008, 18(1): 171-186

25 Grosso A. Finding maximin Latin hypercube designs by iterated local search heuristics. Eur J Oper Res, 2009, 197(2): 541-547

26 Eziol T. Influence of parameter estimation uncertainty in Kriging part I-theoretical development. Hydrol Earth Syst Sci, 2001, 5(2): 215223

27 Lophaven S N, Nielsen H B, Sondergaard J. DACE-A Matlab Kriging toolbox, version 2.0. http://www.imm.dtu.dk/ hbn/pub1/TR0212.ps 